\title{
Implementación electrónica basada en FPAA del oscilador caótico de Lorenz
}

\section{FPAA-based electronic Implementation of the Lorenz chaotic oscillator}

\author{
SILVA-JUÁREZ, Alejandro†*, ESTEVEZ-CARREÓN, Jaime, PONCE-MELLADO, Juan Jorge y \\ HERRERA-SÁNCHEZ, Gustavo
}

Universidad Tecnológica de Puebla

Tecnológico Nacional de México/Instituto Tecnológico de Puebla

ID $1^{\text {er }}$ Autor: Alejandro, Silva-Juárez / ORC ID: 0000-0001-8473-9803, Research ID Thomson: F-6969-2018, arXiv Author ID: Alejandro_Silva, CVU CONACYT ID: 637028

ID $1^{\text {er }}$ Coautor: Jaime, Estevez-Carreón / ORC ID: 0000-0002-2549-3345, Research ID Thomson: N-7519-2013, CVU CONACYT ID: 200706

ID $2^{\text {do }}$ Coautor: Juan Jorge, Ponce-Mellado / ORC ID: 0000-0003-2186-2868, Research ID Thomson: G-6040-2018, arXiv Author ID: Jorge_Ponce, CVU CONACYT ID: 900658

ID $3^{\text {er }}$ Coautor: Gustavo, Herrera-Sánchez / ORC ID: 0000-0001-5276-5062, Research ID Thomson: F-6595-2018, arXiv Author ID: herreragh, CVU CONACYT ID: 459805

DOI: $10.35429 / J E A .2020 .22 .7 .14 .22$

Recibido: Enero 13, 2020; Aceptado: Marzo 30, 2020

\begin{abstract}
Resumen
Hoy en día, los sistemas caóticos son temas muy interesantes para ingenieros, físicos y matemáticos porque la mayoría de los sistemas físicos reales son inherentemente de naturaleza no lineal. Las primeras implementaciones electrónicas de osciladores caóticos autónomos se desarrollaron utilizando amplificadores operacionales y diodos, distintas referencias detallan implementaciones de circuitos y sistemas caóticos utilizando tecnología de circuitos integrados analógicos, dispositivos discretos como FPGA (Field programmable gate arrays), microcontroladores, etc. Sin embargo, las implementaciones analógicas sufren el problema de sensibilidad de los valores de los componentes analógicos y las implementaciones digitales sufren el problema de la degradación debido al número reducido de bits para realizar operaciones aritméticas de computadora. Los sistemas de ecuaciones diferenciales que modelan los osciladores caóticos requieren integradores que pueden implementarse con FPAA's (Field programmable analogue array), en este trabajo se desarrollan implementaciones electrónicas que se miden en condiciones de laboratorio para observar atractores caóticos experimentales, los cuales serán utilizados en la implementación de generadores de números aleatorios y sistemas de comunicación segura para el cifrado de imágenes.
\end{abstract}

Caos, FPAA, Osciladores, Implementación

\begin{abstract}
Nowadays, chaotic systems are very interesting topics for engineers, physicists and mathematicians because most real physical systems are inherently non-linear in nature. The first electronic implementations of autonomous chaotic oscillators were developed using operational amplifiers and diodes, different references detail implementations of chaotic circuits and systems using analog integrated circuit technology, discrete devices such as FPGA (Field programmable gate arrays), microcontrollers, etc. However, analog implementations suffer the problem of sensitivity of analog component values and digital implementations suffer the problem of degradation due to the reduced number of bits to perform computer arithmetic operations. The systems of differential equations that model the chaotic oscillators require integrators that can be implemented with FPAA's (Field programmable analogue array), in this work electronic implementations are developed that are measured in laboratory conditions to observe experimental chaotic attractors, which will be used in the implementation of random number generators and secure communication systems for image encryption.
\end{abstract}

Citación: SILVA-JUÁREZ, Alejandro, ESTEVEZ-CARREÓN, Jaime, PONCE-MELLADO, Juan Jorge y HERRERASÁNCHEZ, Gustavo. Implementación electrónica basada en FPAA del oscilador caótico de Lorenz. Revista de Aplicaciones de la Ingeniería. 2020. 7-22: 14-22.

\footnotetext{
*Correspondencia al Autor (Correo Electrónico: alejandro.silva@utpuebla.edu.mx)

$\uparrow$ Investigador contribuyendo como primer autor.
} 


\section{Introducción}

Los sistemas caóticos se conocen desde hace mucho tiempo, pero solo recientemente se demostró que el caos podía controlarse y, por lo tanto, sincronizarse [1-6]. Por esta razón; Esta clase de sistemas promete tener un gran impacto en muchas aplicaciones novedosas, críticas en términos de tiempo y energía, tales como circuitos electrónicos y dispositivos de alto rendimiento (p. ej., moduladores deltasigma y convertidores de potencia), mezcla de líquidos, reacciones químicas, sistemas biológicos (p. ej. en el cerebro humano, el corazón y el proceso de percepción), la gestión de crisis (por ejemplo, en electrónica de potencia), el procesamiento seguro de la información y la toma de decisiones críticas en eventos políticos, económicos y militares [710]. Esta nueva y desafiante área de investigación y desarrollo se ha convertido en una interdisciplina científica, que involucra ingenieros de sistemas y control, físicos teóricos y experimentales, matemáticos aplicados, fisiólogos y, sobre todo, especialistas en circuitos y dispositivos.

Hoy en día, los generadores de caos han recibido considerable atención para modelarlos, simularlos, diseñarlos y usarlos en aplicaciones de ingeniería. El interés está en los problemas teóricos y prácticos, mientras que una aplicación directa está en el campo de la sincronización caótica para implementar esquemas de comunicación privada, donde la información confidencial que se transmite se incrusta en una señal caótica mediante modulación directa, enmascaramiento u otra técnica $[11,12]$. En el extremo del receptor, si la sincronización caótica se logra perfectamente, se puede extraer la información oculta de la señal transmitida. En este trabajo se realiza el análisis de estabilidad del sistema de Lorenz, las aproximaciones, simulaciones para su implementación en FPAA y utilizarlo en aplicaciones de comunicación segura en transmisión de imágenes.

\section{Sistemas dinámicos no lineales}

Uno de los sistemas de ecuaciones diferenciales no lineales que presenta un comportamiento caotico es el sistema de Lorenz [13], que es descrito por:

$$
\left\{\begin{array}{l}
\dot{x}=\sigma(y-x), \\
\dot{y}=x(\rho-z)-y, \\
\dot{z}=x y-\beta z,
\end{array}\right.
$$

Con parámetros $(\sigma, \rho, \beta)=$ $(10,28,8 / 3)$, para el análisis de estabilidad de los sistemas caoticos se lleva a cabo la siguiente metodología:

- Obtener los puntos de equilibrio.

- Aplicar la matriz jacobiana.

- Calcular los valores propios.

- Determinar la estabilidad del Sistema.

- Determinar el tamaño de paso h.

- Resolver el sistema con el método numérico.

- Calcular las métricas: Exponente de Lyapunov, entropía, dimensión fractal.

El sistema de Lorenz tiene tres puntos de equilibrio; el primero se encuentra en el origen $E_{1}=(0,0,0), \mathrm{y}$ los otros dos estan en $E_{2}=(\sqrt{(\beta(\rho-1)}, \sqrt{(\beta(\rho-1)}, \rho-1), \quad \mathrm{y}$ $E_{3}=(-\sqrt{(\beta(\rho-1)},-\sqrt{(\beta(\rho-1)}, \rho-1)$.

La matriz jacobiana al sistema (1) en los puntos de equilibrio $E_{1}, E_{2}$ y $E_{2}$ esta dada por:

$J=\left(\begin{array}{lll}\frac{\partial f_{1}}{\partial x} & \frac{\partial f_{1}}{\partial y} & \frac{\partial f_{1}}{\partial z} \\ \frac{\partial f_{2}}{\partial x} & \frac{\partial f_{2}}{\partial y} & \frac{\partial f_{2}}{\partial z} \\ \frac{\partial f_{3}}{\partial x} & \frac{\partial f_{3}}{\partial y} & \frac{\partial f_{3}}{\partial z}\end{array}\right)=\left[\begin{array}{ccc}-\sigma & \sigma & 0 \\ \rho-z & -1 & -x \\ y & x & -\beta\end{array}\right]$

La ecuación característica tiene la forma $|\lambda I-J|=0$, se obtienen los valores propios del sistema (1) y resolviendo en los puntos de equilibrio se obtienen los valores propios como sigue:

$$
\left[\begin{array}{lll}
\lambda & 0 & 0 \\
0 & \lambda & 0 \\
0 & 0 & \lambda
\end{array}\right]-\left[\begin{array}{ccc}
-\sigma & \sigma & 0 \\
\rho-z & -1 & -x \\
y & x & -\beta
\end{array}\right]
$$




$$
=\left[\begin{array}{ccc}
\lambda+\sigma & -\sigma & 0 \\
-\rho & \lambda+1 & -x \\
-y & -x & \lambda+\beta
\end{array}\right]
$$

$E_{1}=\lambda_{1} \approx-13.8546, \lambda_{2,3} \approx 0.0940 \pm 10.1945 i$

$E_{2}=\lambda_{1} \approx-13.8546, \lambda_{2,3} \approx 0.0940 \pm 10.1945 i$

$E_{3}=\lambda_{1} \approx-22.82776, \lambda_{2} \approx 11.8277, \lambda_{3} \approx-2.6667$

En la Figura 1 se muestran las simulaciones del sistema de Lorenz, donde se muestra la creación de un oscilador caótico sensible a las condiciones iniciales aplicando el método de integración de Runge-Kutta de cuarto orden [14]. Cada aproximación numérica se ha realizado con tamaño de paso de integración $h=0.001$, tiempo de simulación $t_{\text {sim }}=100$ segundos $\mathrm{y}$ condiciones iniciales $\mathrm{x}(0)=\mathrm{y}(0)=\mathrm{z}(0)=0.1$, los algoritmos se programaron con Matlab R2019a ® en doble precisión, el tipo de error por redondeo y épsilon de máquina de 2.2204e-16. Dados los resultados de los puntos de equilibrio y los valores propios, se verifica que, basándose en los signos de los valores propios y si corresponden a reales o imaginarios, se observa que todos los sistemas presentan inestabilidad de tipo asintótica, nodo silla, foco silla, las cuales son características necesarias para generar un comportamiento caótico.
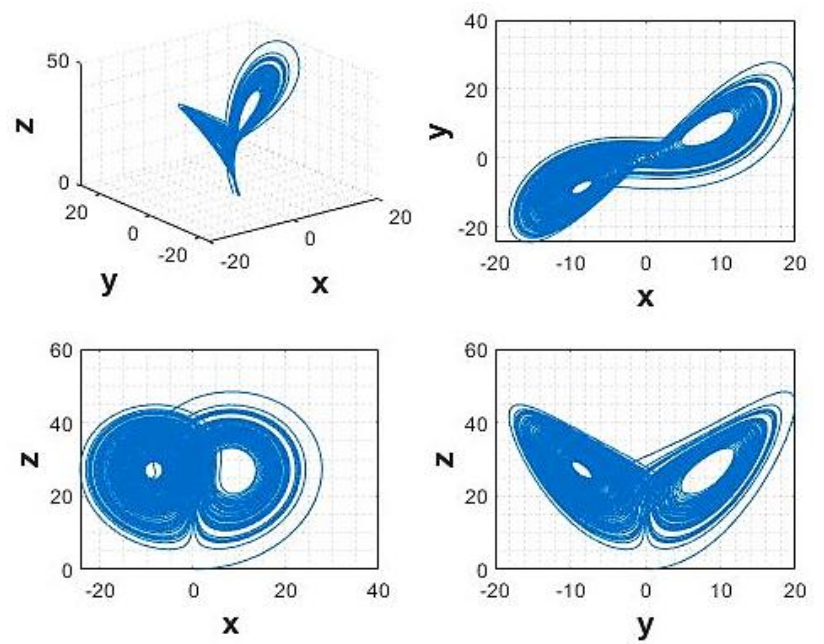

Figura 1 Resultado de la simulación numérica del sistema de Lorenz en espacio de estados en los planos $3 \mathrm{D}, \mathrm{xy}, \mathrm{xz}$ y yz respectivamente

\section{Implementación analógica}

La realización electrónica del sistema de Lorenz puede realizarse con amplificadores operacionales y elementos pasivos $\mathrm{R}-\mathrm{C}$, el procedimiento de diseño comienza analizando las series de tiempo de las variables $x, y$ y $z$ del sistema (1) como se observa en la Figura 2.
Es importante considerar para efectos de implementación que la dinámica de las tres variables de estado, tienen rangos de amplitudes $(-21.5 ; 21.5)$ para $x,(-29.4 ; 29.4)$ para $y$ y $(-54.4 ; 54.4)$ para $z$, es importante notar que estos rangos exceden los valores de salida de voltaje de los amplificadores que normalmente son $\pm 12 \mathrm{~V}$.

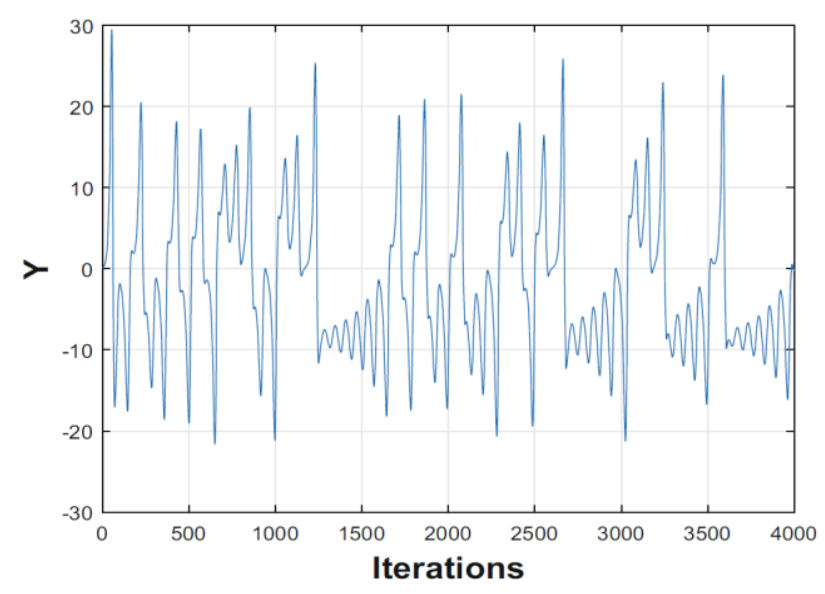

a)

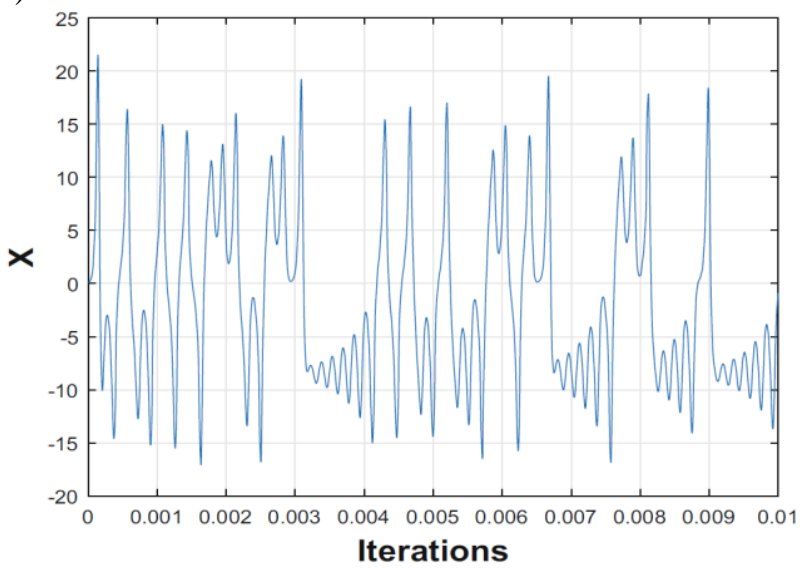

b)

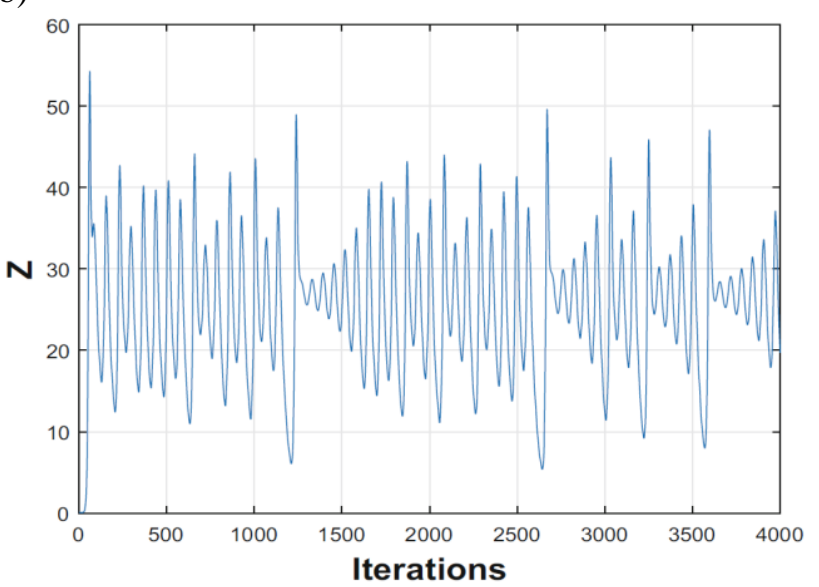

c)

Figura 2 Series de tiempo vs amplitudes de las variables de estado del atractor de Lorenz: a) $\mathrm{x}, \mathrm{b}$ ) y c) $\mathrm{z}$

SILVA-JUÁREZ, Alejandro, ESTEVEZ-CARREÓN, Jaime, PONCEMELLADO, Juan Jorge y HERRERA-SÁNCHEZ, Gustavo. Implementación electrónica basada en FPAA del oscilador caótico de Lorenz. Revista de Aplicaciones de la Ingeniería. 2020 
Una implementación del sistema (1) se muestra en las Figuras 3, 4 y 5, el circuito analógico requiere de tres amplificadores operacionales (TL082) donde, cada uno realiza la operación de sumador, restador e integrador, y dos multiplicadores analógicos AD633 para implementar los términos no lineales en las ecuaciones del circuito formados por los productos de las variables de estado $x z$ y $y z$.

Implementar directamente el sistema de ecuaciones de (1) en su forma original en un circuito analógico implica un escalamiento, ya que las variables de estado ocupan un rango dinámico con valores que exceden los límites del voltaje de alimentación típica de un OPAMP $\pm 12 \mathrm{~V}$ como se puede observar en la Figura 2. Específicamente, para los coeficientes $\sigma, \rho$ y $\beta$ los valores de las resistencias se escalan a $1 \mathrm{M} \Omega$, por lo tanto, la representación del bloque de la primera ecuación $\dot{x}=\sigma(y-x)$ se tiene:

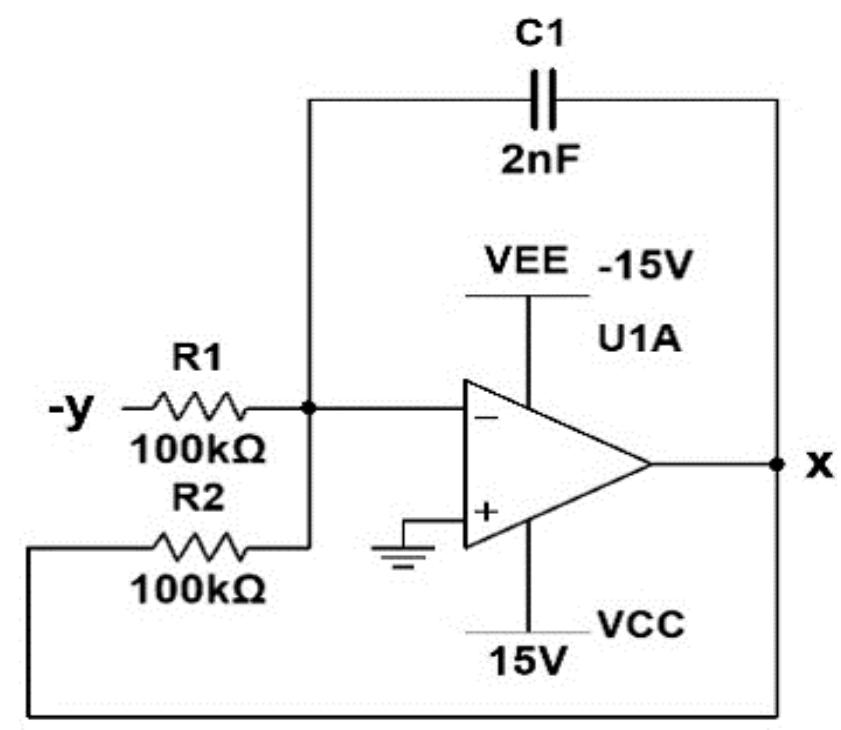

Figura 3 Circuito para la variable $\mathrm{x}$ de (1)

Donde $\sigma=10$ esta asociada al valor de $\begin{array}{llllllll}R_{1} & \text { y } & R_{2} & \text { para } & \text { las variables } & x & \mathrm{y} & z\end{array}$ respectivamente. Si se define el valor de $C_{1}=$ $C_{2}=C_{3}=2 \mathrm{nf}$, el valor escalado de $R_{1}=$ $1 \mathrm{M} \Omega / 100 \mathrm{k} \Omega$ y la constante de tiempo para los tres integradores se calcula como $1 / R_{1} C_{1}=$ $5 \times 10^{3}$ como se observa en la figura 3.

La segunda ecuación de (1) $\dot{y}=$ $x(\rho-z)-y$, se implementa en la figura 4 , donde $\rho=28$ esta asociada al valor de $R_{3}$ y la variable $x$, el valor escalado de $R_{3}=$ $11 \mathrm{M} \Omega / 35.7 \mathrm{k} \Omega$.
La segunda entrada está relacionada con la salida $-x z$ del multiplicador analógico AD633 y $R_{3}$ con escalamiento unitario, las ecuaciones en el diagrama están normalizadas a $0.1 \mathrm{~V}$, de ahí el factor de escala multiplicador de 100 , es decir $R_{4}=1 \mathrm{M} \Omega / 10 \mathrm{k} \Omega$, por último, la tercera entrada tiene como variable a $y$ con valor unitario $R_{5}=1 \mathrm{M} \Omega / 1 \mathrm{M} \Omega$.

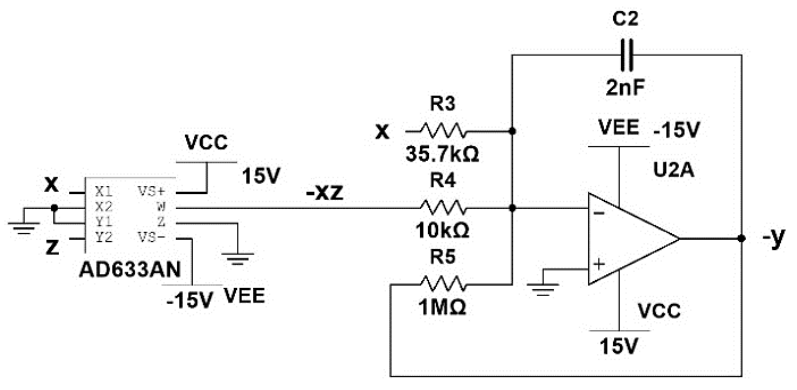

Figura 4 Circuito para la variable y de (1)

El tercer bloque de la ecuacion $\dot{z}=$ $x y-\beta z$, se muestra en la figura 5 donde se relaciona la salida $-x y$ del multiplicador analógico $\mathrm{AD633}$ y $R_{6}$ con escalamiento unitario, es decir $R_{5}=1 \mathrm{M} \Omega / 10 \mathrm{k} \Omega$, finalmente la segunda entrada tiene como variable a $z$ y su coeficiente $\beta=8 / 3$, es decir $R_{7}=1 \mathrm{M} \Omega / 374 \mathrm{k} \Omega$.

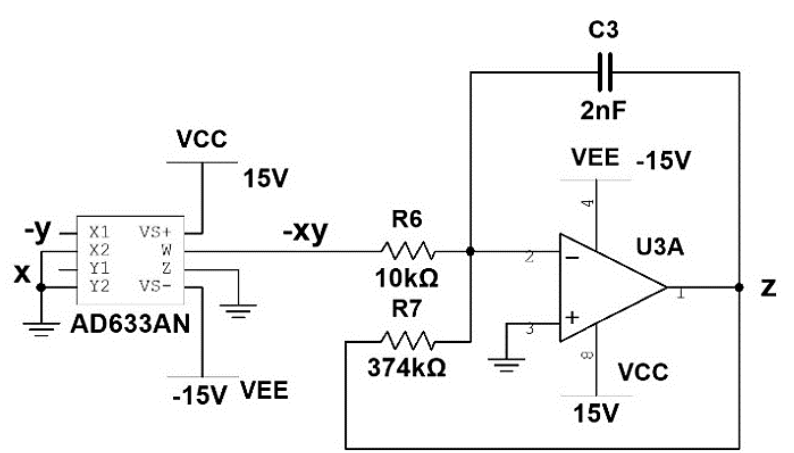

Figura 5 Circuito para la variable $\mathrm{z}$ de (1)

Con este escalamiento del sistema (1), todas las variables de estado tienen un rango dinámico similar y los voltajes del circuito se mantienen dentro del rango de los límites de alimentación típicos $\pm 12 \mathrm{~V}$. El conjunto de ecuaciones de estado que gobiernan el comportamiento dinámico del circuito esta dado por (2), valores de los componentes pasivos son $R_{1}=R_{2}=100 \mathrm{k} \Omega, R_{3}=35.7 \mathrm{k} \Omega$, $R_{4}=R_{6}=10 \mathrm{k} \Omega, R_{5}=1 \mathrm{M} \Omega, R_{7}=374 \mathrm{k} \Omega \mathrm{y}$ $C_{1}=C_{2}=C_{3}=2 \mathrm{nF}$.

$\frac{d x}{d t}=-\frac{1}{R_{1} C_{1}} y+\frac{1}{R_{2} C_{2}} x$ 
$\frac{d y}{d t}=\frac{1}{R_{3} C_{2}} x-\frac{1}{R_{4} C_{2}} x z \frac{1}{R_{5} C_{2}} y$

$\frac{d z}{d t}=-\frac{1}{R_{6} C_{3}} x y+\frac{1}{R_{7} C_{3}} z$

Construyendo los circuitos de las figuras 3,4 y 5 y uniendo las etiquetas de las variables, se obtiene la implementación del circuito analógico del sistema de Lorenz, los resultados de la simulación en multisim muestran la creación del atractor en la Figura $6(a, b, c)$.

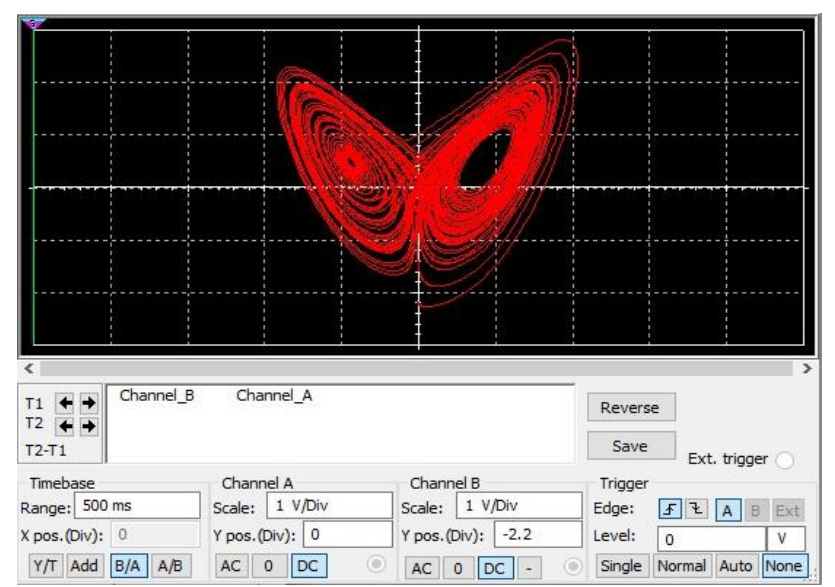

a)
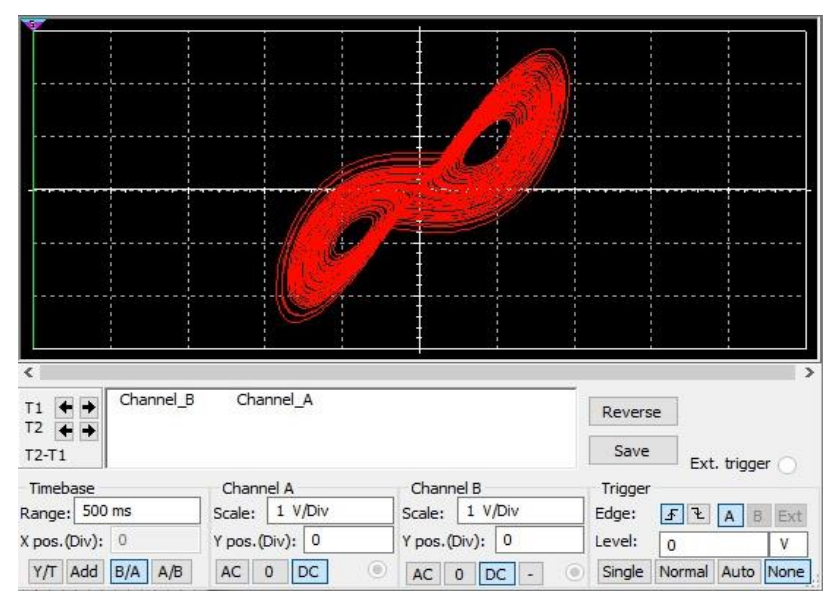
b)
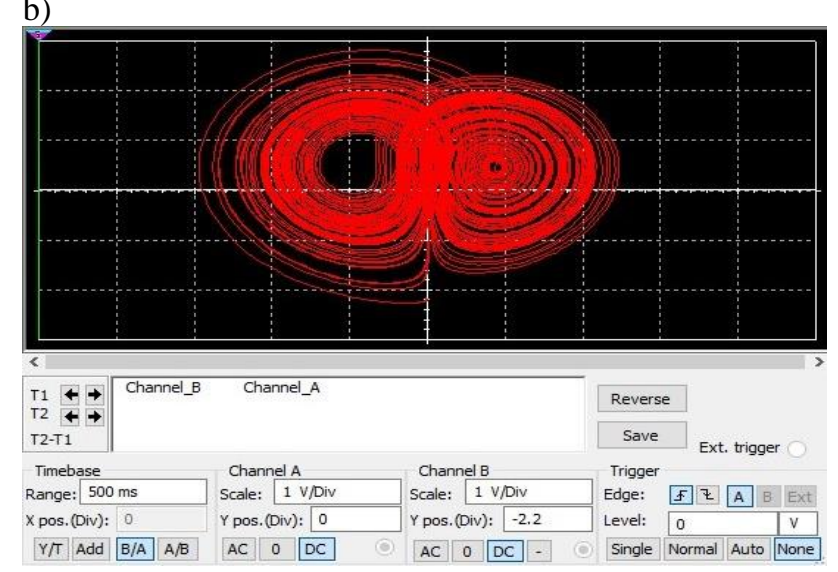
c)

Figura 6 Atractores caóticos del sistema de Lorenz (1V/Div): a) plano xy, b) plano xz, c) yz

\section{Implementación usando el FPAA AN231E04}

Un arreglo análogo programable FPAA (Field Programmable Analog Array) es el equivalente al arreglo programable digital FPGA (Field Programmable Gate Array) en cuanto a su organización interna, pero, su gran diferencia es que su aplicación es exclusivamente para procesamiento análogo. Un FPAA se puede usar para implementar una amplia variedad de funciones analógicas, como la integración, derivación, suma/resta ponderada, filtrado, rectificación, comparador, multiplicación, división, conversión de analógico a digital, referencias de voltaje, acondicionamiento de señal, amplificación, síntesis de funciones no lineales y generación de señales arbitrarias, entre otras [15].

Para la implementación en FPAA del sistema (1), se utiliza la tarjeta Anadigm QuadApex la cual costa de cuatro Chips AN231E04 y maneja un voltaje de salida máximo de $\pm 3 \mathrm{~V}$, como es evidente, es necesario reescalar el rango dinámico de las variables de estado ya que exceden los valores de salida de los chips, para esto se eligen los siguientes factores de reescalamiento:

$\left\{\begin{array}{l}X=\frac{x}{k_{x}} \\ Y=\frac{y}{k_{y}} \\ Z=\frac{z}{k_{z}}\end{array}\right.$

con $k_{x}=5, k_{y}=5, k_{z}=5$. Al aplicar este cambio de escala al sistema de Lorenz se obtiene:

$\dot{X}=\sigma\left(\frac{k_{y}}{k_{x}} Y-X\right)$

$\dot{Y}=\rho \frac{k_{x}}{k_{y}} X-Y-\frac{k_{x} k_{z}}{k_{y}} X Z$

$\dot{Z}=\frac{k_{x} k_{y}}{k_{z}} X Y-\beta Z$

Todos los parámetros del circuito son programables y sus valores no se pueden fijar con precisión, se implementan con algunos parámetros de tolerancia. Además, es necesario introducir parámetros adicionales en el sistema (4) para superar las imprecisiones debidas al dispositivo programable, por lo que el modelo implementado en el dispositivo analógico se puede escribir de la siguiente manera: 


$$
\begin{aligned}
& \dot{X}=\alpha\left(\frac{k_{y}}{k_{x}} Y-X\right) \\
& \dot{Y}=G_{1} \rho \frac{k_{x}}{k_{y}} X-G_{2} Y-G_{3} \frac{k_{x} k_{z}}{k_{y}} X Z \\
& \dot{Z}=G_{4} \frac{k_{x} k_{y}}{k_{z}} X Y-G_{5} \beta Z
\end{aligned}
$$

Los parámetros $G_{1}, G_{2}, G_{3}, G_{4}$ y $G_{1}$ se ajustan experimentalmente para compensar estas inexactitudes. Además de los multiplicadores, el elemento que realiza las sumas de entradas y las integraciones del sistema es la CAM Sum/Difference Integrator. Esta CAM crea un integrador sumador con hasta tres entradas, las entradas pueden ser invertidas o no invertidas, de modo que se pueden crear sumas y diferencias en la función de transferencia. Cada rama de entrada muestreada tiene una constante de integración programable. La funcion de transferencia para este circuito es

$$
\frac{\Delta V_{\text {out }}}{\Delta t}= \pm K_{1} V_{\text {Input } 1} \pm K_{2} V_{\text {Input } 2} \pm K_{3} V_{\text {Input } 3}
$$

Las variables $K$ son las constantes de integración de las diversas entradas y las variables de entrada $V$ son los voltajes de entrada en las diversas entradas. El signo de cada término depende de la polaridad seleccionada para cada rama de entrada en las Opciones CAM. Los términos se agregan para las entradas no inversoras y se restan para las entradas inversoras. Por ejemplo, la función de transferencia para una CAM configurada con solo dos entradas no inversoras es: $\Delta V_{\text {out }} / \Delta t=$ $\pm K_{1} V_{\text {Input } 1} \pm K_{2} V_{\text {Input } 2}$, donde $\Delta V_{\text {out }}$ es el cambio en el voltaje de salida durante un período del reloj cuando $\Delta \mathrm{t}$ es la duración de un período de reloj [15]. La función de transferencia equivalente en el dominio $s$ es:

$V_{\text {out }}(s)=\frac{ \pm K_{1} V_{\text {Input } 1}(s) \pm K_{2} V_{\text {Input } 2}(s) \pm K_{3} V_{\text {Input } 3}(s)}{s}$

Los valores de los capacitores se eligen en función de las siguientes relaciones: : $K_{1}=$ $\frac{f_{C} C_{i n A}}{C_{i n t}}, K_{2}=\frac{f_{C} C_{i n B}}{C_{i n t}}$, y $K_{3}=\frac{f_{C} C_{i n C}}{C_{i n t}}$.

Las constantes de integración se calculan de la siguiente manera: $K=1 / R C$. Se debe tomar en cuenta también que en Anadigm Designer 2 las constantes del integrador están en unidades de $1 / \mu S$, no $1 / S$.
Entonces, cuando se calcula $K$ es $K=1 \times 10^{-6} / R C$ para obtener los parámetros CAM como se muestra en la tabla 3. En la Fig 18 las IOCELL 5, 6 y 7 estan configuradas en modo salidas y tipo Bypass. Otra de las CAM utilizadas para realizar el diseño analógico son las operaciones de multiplicación de las variables $x$ y $z$ en la segunda y $x$ y $y$ en la tercera ecuación del sistema 1, estas son implementadas por las CAM Multiplier que tienen la siguiente función de transferencia: $V_{\text {out }}=M \cdot V_{x} \cdot V_{y}$, donde $M$ es el factor de multiplicación, esta CAM de dos entradas requiere de dos frecuencias; Clock A y Clock $\mathrm{B}$ donde la relación debe ser 16 veces Clock B respecto al Clock A [16]. En la tabla 1 se muestra la configuración del FPAA para todos los Clock's a utilizar en la implementación del sistema (1).

\begin{tabular}{|l|l|}
\hline Master clock- ACLK $(\mathrm{fc})$ & $16 \mathrm{MHz}$ \\
\hline Systems clock $1(\mathrm{sys} 1=\mathrm{fc} / 20)$ & $800 \mathrm{kHz}$ \\
\hline Systems clock $2(\mathrm{sys} 2=\mathrm{fc} / 1)$ & $16 \mathrm{MHz}$ \\
\hline Clock 0 (sys1/1) & $800 \mathrm{kHz}$ \\
\hline Clock 1 (sys1/16) & $50 \mathrm{kHz}$ \\
\hline
\end{tabular}

Tabla 1 Frecuencias de los Clocks en el FPAA

El sistema (1) es autónomo y no requiere de entradas o señales externas para generar su comportamiento dinámico, sin embargo, para realizar la simulación utilizando Anadigm Designer 2 se requiere de un generador de señal.

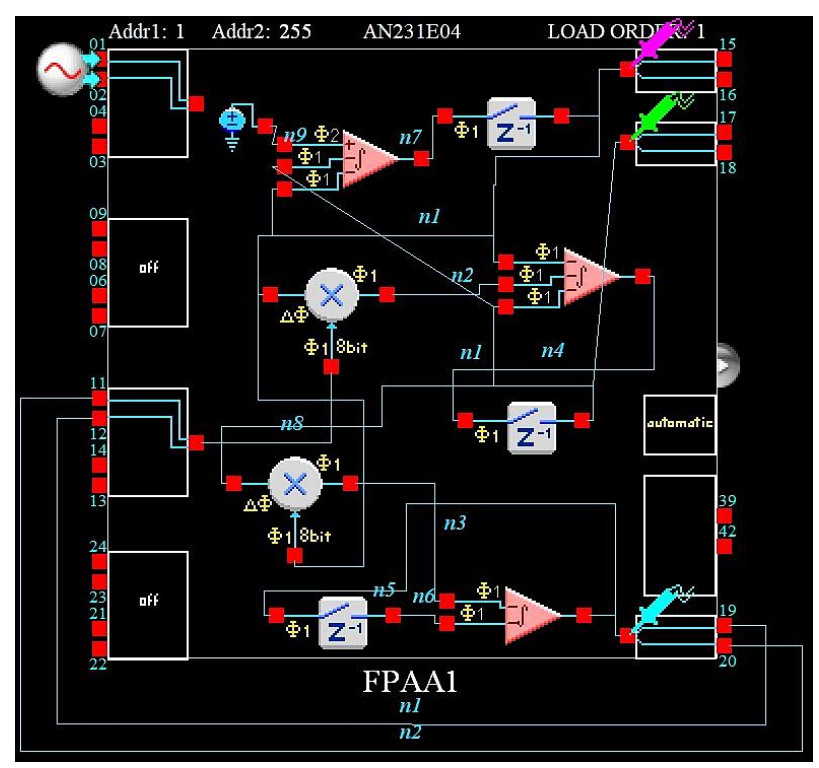

Figura 7 Descripción del oscilador caotico de Lorenz en Anadigm Designer 2 incluyendo bloques de retardo $\left(\mathrm{z}^{-1}\right)$ para observar las variables de estado $\mathrm{x}, \mathrm{y}, \mathrm{z}$ 
En la figura 7 se observa que tal generador de señal no realiza ninguna función, se añade al circuito solo con el propósito de que el software no indique error. El propósito de las CAM de retención es agregar retrasos $\left(z^{-1}\right)$ de una fase de reloj [15] ya que con esto se evitan bucles de retardo cero en el circuito, esto es debido a que mientras la tarjeta FPAA trabaja con señales analógicas, el simulador para fines de mostrar la señal, requiere tratarla como digital. Se puede ver que se realizó una conexión externa entre las salidas 007 y la entrada IO3, observando con atención esta conexión se verá que los cables están intercambiados, el propósito de esta conexión es simplemente invertir la señal ya que no se tienen suficientes recursos dentro de FPAA para agregar un inversor $y$ mostrar la simulación.

Es importante mencionar que cuando se implementa el circuito en el FPAA no es necesario usar las CAM de retención $\left(z^{-1}\right)$. En la figura $8 \mathrm{a}$ y $8 \mathrm{~b}$ se muestran las series de tiempo de las variables de estado $x, y, z$ en el simulador Anadigm Designer 2, también se observa que la amplitud del voltaje de salida no excede los rangos de voltaje del chip AN231E04 que es de $\pm 3 \mathrm{~V}$, así como las características de no linealidad y no periodicidad que son condiciones necesarias para generar un comportamiento caótico.

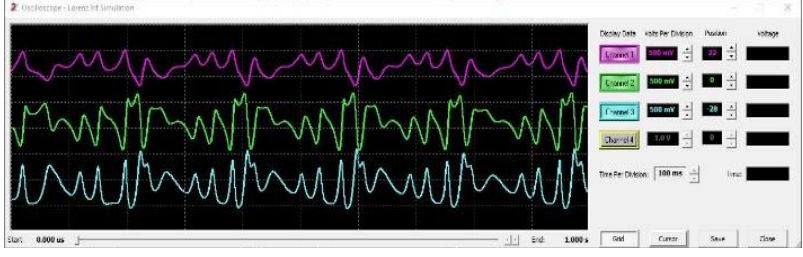

a)

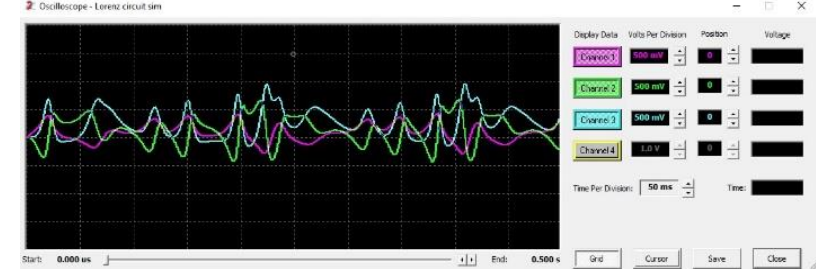

b)

Figura 8 Atractores caóticos del sistema de Lorenz (1V/Div): a) plano $x y, b$ ) plano $x z, c) y z$

\begin{tabular}{|c|c|c|c|}
\hline laque & 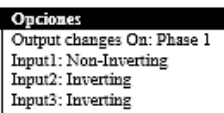 & 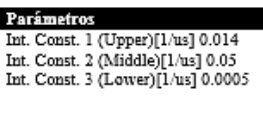 & $\begin{array}{l}\text { Clocks } \\
\text { ClockA 50 kHz } \\
\text { (Chip Clock 1) }\end{array}$ \\
\hline & 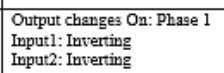 & 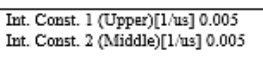 & \begin{tabular}{|l}
$\begin{array}{l}\text { ClockA 50 kHz } \\
\text { (Chip Clock 1) }\end{array}$ \\
\end{tabular} \\
\hline & 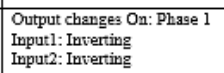 & 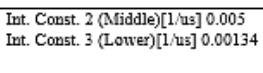 & \begin{tabular}{|l}
$\begin{array}{l}\text { ClockA } 50 \text { kHiz } \\
\text { (Chip Clock 1) }\end{array}$ \\
\end{tabular} \\
\hline & \begin{tabular}{|l|} 
Output changes On: Phase 1 \\
Sample and Hold: Off
\end{tabular} & Multiglication Factor 1.00 & 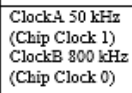 \\
\hline & $\begin{array}{l}\text { Output changes On: Phase 1 } \\
\text { Smple and Hold: Off }\end{array}$ & Multiglication Factor 1.00 & 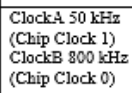 \\
\hline & \begin{tabular}{|l} 
Input Sampling Phase 1 \\
Sample and Hold: Off
\end{tabular} & Gain 1.00 & \begin{tabular}{|l}
$\begin{array}{l}\text { ClockA } 50 \mathrm{kHz} \\
\text { (Chip Clock 1) }\end{array}$ \\
\end{tabular} \\
\hline & \begin{tabular}{|l} 
Input Sampling Phase 1 \\
Sample and Hold: Off
\end{tabular} & Gain 1.00 & \begin{tabular}{|l} 
ClockA $50 \mathrm{kHz}$ \\
(Chip clock 1)
\end{tabular} \\
\hline OCELL5 & \begin{tabular}{|l} 
IO Mode: Output \\
Output of variable $x, y, z$
\end{tabular} & Output Type: Bypass & \\
\hline
\end{tabular}

Tabla 2 Módulos analógicos configurables del FPAA para la implementación del sistema Lorenz en la tarjeta Anadigm QuadApex con Chip AN231E04

La implementación del sistema (1) en la FPAA Anadigm QuadApex Development Board con chip AN231E04 requiere de la configuración de las CAM mostradas en la tabla 2, en la figura 9 se muestra el esquema del circuito implementado y finalmente en la figura 10 se muestran los resultados en osciloscopio de la implementación del sistema de Lorenz con el atractor en los planos $x y, x z$ y $y z$ respectivamente a 50mV/Div en osciloscopio, además en la figura $10 \mathrm{~d}$ se observa la serie de tiempo de las variables $x$ a $200 \mathrm{mV} / \mathrm{Div}$ con una amplitud de $1.518 \mathrm{~V}$ y $y$ a $50 \mathrm{mV} / \mathrm{Div}$ con una amplitud de $1.666 \mathrm{~V}$ respectivamente.

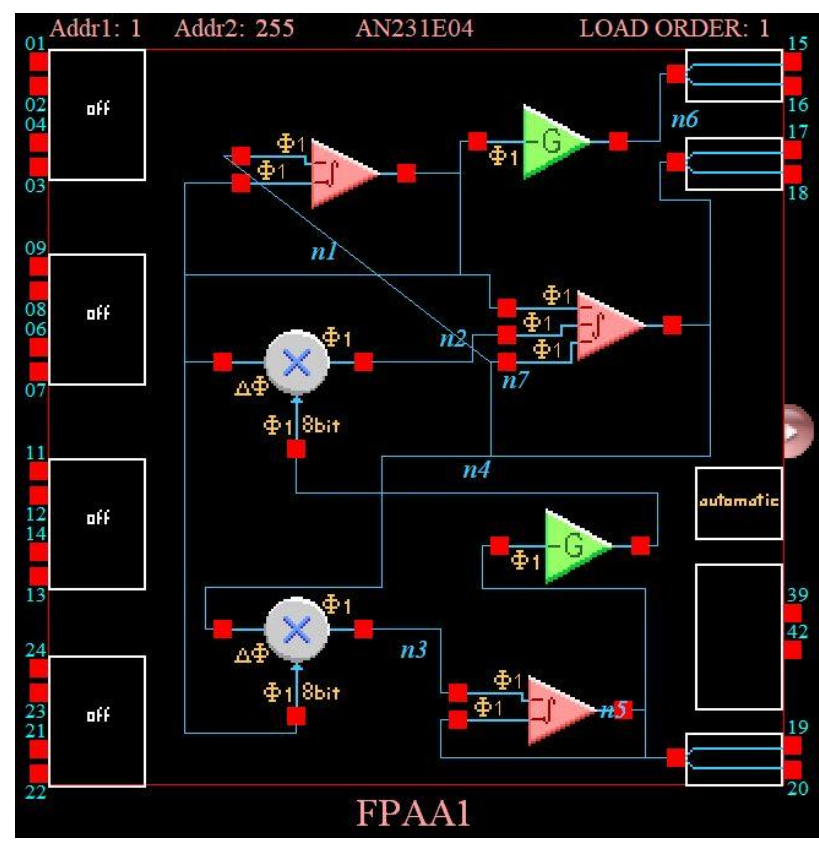

Figura 9 Implementación electrónica analógica del sistema de Lorenz en FPAA

SILVA-JUÁREZ, Alejandro, ESTEVEZ-CARREÓN, Jaime, PONCEMELLADO, Juan Jorge y HERRERA-SÁNCHEZ, Gustavo. Implementación electrónica basada en FPAA del oscilador caótico de Lorenz. Revista de Aplicaciones de la Ingeniería. 2020 

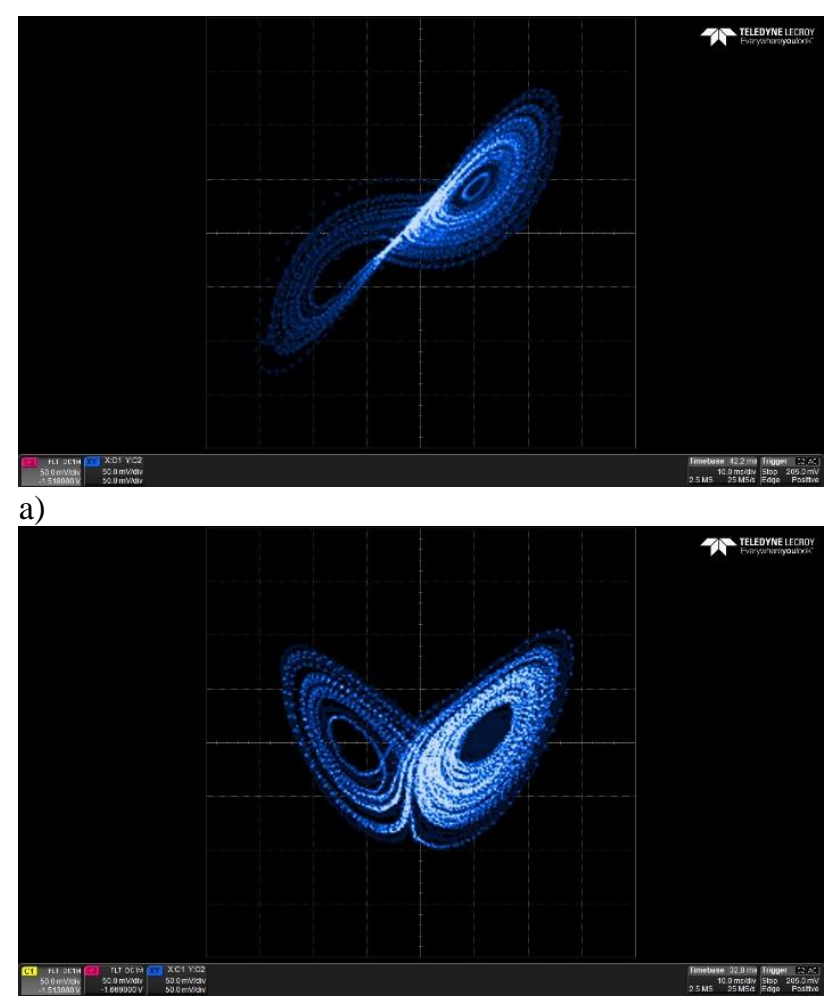

b)

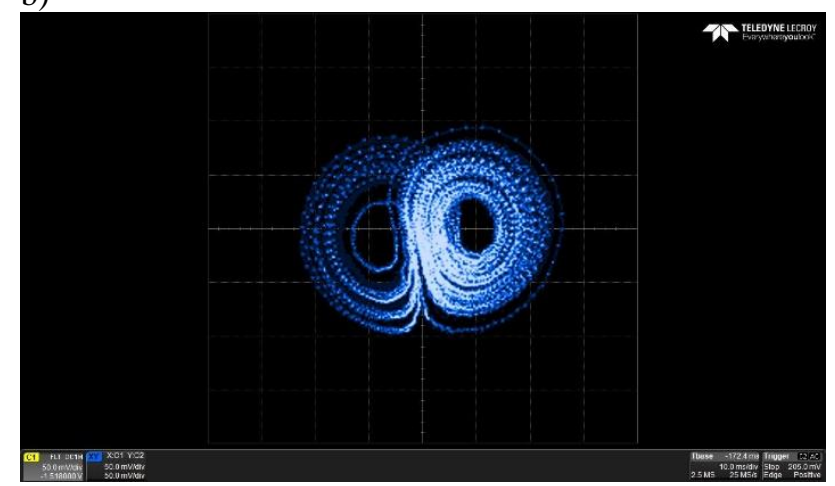

c)

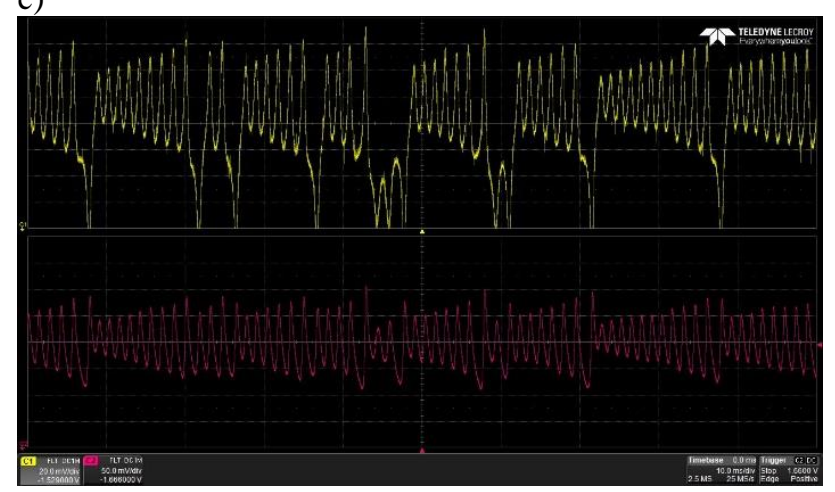

d)

Figura 10 Atractores caóticos del sistema de Lorenz (50 mV/Div): a) plano xy, b) plano xz, c) yz y d) Series de tiempo de $\mathrm{x}$ y $\mathrm{y}$

\section{Conclusiones}

Se ha presentado la implementación programable basada en FPAA del sistema caótico de Lorenz que es adecuado para un diseño reconfigurable de acuerdo con su dinámica autónoma no lineal.
Los resultados experimentales concuerdan con los resultados obtenidos de experimentos establecidos por componentes discretos como los FPGA. Este sistema puede usarse efectivamente como un generador de caos programable en muchas aplicaciones basadas en el caos, como la sincronización de sistemas maestro-esclavo para transmisión de imagen, criptografía y seguridad en comunicaciones.

\section{Referencias}

[1] T. Parker and L. Chua, "The dual double scroll equation," IEEE Transactions on Circuits and Systems, vol. CAS-34, no. 9, pp. 1059-1073, 1987.

[2] . M. Pecora and T. L. Carroll, "Synchronization in chaotic systems," Physical review letters, vol. 64, no. 8, p. 821, 1990.

[3] K. M. Cuomo and A. V. Oppenheim, "Circuit implementation of synchronized chaos with applications to communications," Physical review letters, vol. 71, no. 1, p. 65, 1993.

[4] S. Yu, Z. Ma, S. Qiu, S. Peng, and Q. Lin, "Generation and synchronization of nscroll chaotic and hyperchaotic attractors in fourthorder systems," Chin. Phys., no. 13, pp. 317-328, 2004.

[5] P. Stavroulakis, Chaos applications in telecommunications. CRC press, 2005.

[6] Kapitaniak, Chaos for engineers: theory, applications, and control. Springer Science \& Business Media, 2012.

[7] Silva-Juarez, A., Rodriguez-Gomez, G., de la Fraga, L. G., Guillen-Fernandez, O., \& Tlelo-Cuautle, E. (2019). Optimizing the kaplan-yorke dimension of chaotic oscillators applying de and pso. Technologies, 7(2), 38.

[8] Tlelo-Cuautle, E., Pano-Azucena, A. D., Guillén-Fernández, O., \& Silva-Juárez, A. (2020). Integer and Fractional-Order Chaotic Circuits and Systems. In Analog/Digital Implementation of Fractional Order Chaotic Circuits and Applications (pp. 1-40). Springer, Cham. 
[9] Silva-Juárez, A., Morales-Pérez, C. J., de la Fraga, L. G., Tlelo-Cuautle, E., \& de Jesús Rangel-Magdaleno, J. (2019). On maximizing the positive Lyapunov exponent of chaotic oscillators applying DE and PSO. International Journal of Dynamics and Control, 7(4), 11571172.

[10] H. Degn, A. V. Holden, and L. F. Olsen, Chaos in biological systems. Springer Science \& Business Media, 2013, vol. 138.

[11] R. Weller, "Resistance, chaos and control in china: Taiping rebels, taiwanese ghosts and tiananmen," 2013.

[12] S. H. Strogatz, Nonlinear dynamics and chaos: with applications to physics, biology, chemistry, and engineering. Westview press, 2014.

[13] Curry, J. H. (1978). A generalized Lorenz system. Communications in Mathematical Physics, 60(3), 193-204.

[14] Chapra, S. C., \& Canale, R. P. (2010). Numerical methods for engineers. Boston: McGraw-Hill Higher Education.

[15] Anadigm, Dynamically Reconfigurable dpASP, 3rd Generation, AN231E04 Datasheet Rev 1.2 www.anadigm.com, 2014 (2014).

[16] Anadigm designer ® R2 user manual, www.anadigm.com (2014) 